\title{
Reflux-nephropathy: modern approaches to diagnostics and treatment
}

\begin{abstract}
The subject of current study is reflux nephropathy (RN) in children with vesicoureteral reflux (VUR) which remains an urgent problem in pediatric nephrology. ${ }^{1}$ According to the European Renal Association-European Dialysis Transplant Association (2010), ${ }^{4}$ the frequency of $\mathrm{RN}$ is up to $22 \%$ (6-27.5\% according to various authors).$^{2-4}$ One of the main pathogenesis factors in the development of tubulointerstitial fibrosis in any form of nephropathy isintraglomerular hypertension. ${ }^{5}$ According to modern concepts of the renin-angiotensin-aldosterone system (RAAS), it is directly involved in the regulation of homeostasis of the body, including maintaining normal kidney function, and its architectonics. When renal tissue is involved in the pathological process (infection, obstruction), then RAAS activation occurs, which leads to activation of mesangial cells and renal tubular apparatus which secrete pro- and anti-inflammatory cytokines and growth factors. ${ }^{6}$
\end{abstract}

Keywords: chronic kidney disease, fibronectin, proteinglikans, myofibroblasts, macrophages, platelets, fibroblasts, tubular epithelial
Volume 7 Issue 4 - 2019

\author{
Natalia Zaicova, ${ }^{1,2,3}$ Vladimir Dlin, ${ }^{2}$ LV \\ Sinitcina, ${ }^{3}$ Anatoly Korsunskii, ${ }^{2}$ NE Revenco ${ }^{3}$ \\ 'Pirogov Russian National Research Medical University, Moldova \\ ${ }^{2}$ Department of Pediatrics and Communicable Diseases, I.M. \\ Sechenov First Moscow State Medical University, Moldova \\ ${ }^{3}$ Institution of Mother and Child Care, Moldova
}

\begin{abstract}
Correspondence: Natalia Zaicova, Department of Pediatrics and Communicable Diseases, I.M. Sechenov First Moscow State Medical University, Moldova, Email nataliazaikova@mail.ru
\end{abstract}

Received: October 08, 2018 | Published: July 30, 2019
Abbreviations: RN, reflux nephropathy; VUR, vesicoureteral reflux; RAAS renin-angiotensin-aldosterone system; CKD, chronic kidney disease; TGF- $\beta 1$, Transforming growth factor- $\beta 1$; AngII, Angiotensin II; VG, van-gizon

\section{Introduction}

The study by the aim of which was to identify markers in the early stages of renal damage revealed that the children with obstruction of the urinary tract or VUR demonstrate specific markers: proteinuria, hypertension, decreased renal functional reserve and disruption of cortical blood flow, according to the color Doppler mapping. The authors also confirmed their significance as early markers for the progression of chronic kidney disease (CKD). ${ }^{7}$ However, according to other authors, these markers are informative for long-term current renal lesions, when the main complications of the renal process developed, and therefore cannot be early and used as prognostic markers. ${ }^{8,9}$ Transforming growth factor- $\beta 1$ (TGF- $\beta 1$ ) is a multifunctional cytokine that regulates normal cell growth and differentiation during embryogenesis, as well as it plays leading role in the development of tissue fibrosis after injury. Their sources are macrophages, platelets, fibroblasts, activated T-lymphocytes, vascular endothelial cells and renal tubules. As a response to damage of renal tissue TGF- $\beta 1$ causes transformation of tubular epithelial cells into myofibroblasts, acting as a potential stimulator of fibroblast proliferation, stimulates production of extracellular matrix proteins (fibronectin, proteinglikans), activates their production and accumulation in the interstitium and accumulation of collagen(types I, III, IV)in the basement membrane. ${ }^{10}$ Angiotensin II (AngII) is a vasoactive protein, as well as a true cytokine that regulates cell growth, proliferation and differentiation of monocytes into macrophages, and also regulates the synthesis of several proinflammatory mediators and growth factors such as TGF- $\beta 1$, which contributes to inflammation and formation of fibrosis. It is known that, as a major factor of profibrogenic initiating and maintaining processes of fibrillogenesis, AngII increases the synthesis of TGF- $\beta 1$, which in turn stimulates cell proliferation and formation of connective tissue. ${ }^{11}$
The purpose of our work was to prove the renoprotective effect of early administration of ACE inhibitors in bacterial obstructive pyelonephritis in rats and the effect of their prolonged use on the urine level of profibrogenic markers.

\section{Experimental part}

In experimental studies, a method of modeling pyelonephritis is known, which is reproduced by introducing a daily culture of Escherichia coli in the amount of 200-500 million of microbial bodies into the lumen of the ureter, followed by its ligation. ${ }^{12} \mathrm{E}$. coli was used as the bacterial culture. The choice of infection is due to the fact that the role of conditional pathogenic flora (lactose-negative enterobacteria, clostridia), in particular, of E. coli, in the development of inflammatory diseases kidneys and urinary tract remains high. As it was shown by experimental studies, the proposed model has changes, characteristic to clinical course of chronic inflammatory diseases in humans. ${ }^{13}$

Characteristics of the experimental groups. The study was performed on 27 adult white male rats of same age, weighing $240 \pm 40$ $\mathrm{g}$ (weight from $200 \mathrm{~g}$ to $260 \mathrm{~g}$ ), the maximum duration of observation was 6 months. According to the goal for the experimental study, the experimental individuals were divided into 3 main groups:

Group $1(n=15)$ - modeling of urethral stenosis, infection of the urinary tract, treatment with antibiotic and ACE inhibitors (enalapril);

Group $2(n=9)$ - modeling of urethral stenosis, infection of urinary tract, antibiotic treatment;

Group $3(n=3)$ - control group.

All experimental individuals were kept in the same conditions of the vivarium, in separate cages, in a well-ventilated room at $20^{\circ} \mathrm{C}$ and fed with standard food. Reproduction of bacterial pyelonephritis in individuals of both groups was carried out by introducing of daily culture of microbial E. coli $(1 \mathrm{ml})$ into the bladder through a catheter $(0.2 \mathrm{~cm}$ in diameter). E. coli obtained from the urine of a sick child with acute pyelonephritis was used as a bacterial culture. Urethral 
stenosis in rats was created by scarifying of urethra with a metal catheter and injecting $1 \mathrm{ml}$ of solution of iodine in ethanol into the lumen of the urethra after anesthesia with $5 \%$ ketamine. ${ }^{14}$ After 48 72 hours of development of the first signs of pyelonephritis, the rats of groups 1 and 2 were given an antibiotic, cefotaxime, once a dose of $100 \mathrm{mg} / \mathrm{kg} / 24 \mathrm{~h}$ intramuscularly for 5 days. The E. coli culture administered to rats was sensitive to this antibiotic according to the results of sowing and determination of antibiotic sensitivity. Enalapril was used only in group 1 of rats at a dose of $0.1 \mathrm{mg} / \mathrm{kg} / 24 \mathrm{~h}(0.024$ $\pm 0.03 \mathrm{mg} / 24 \mathrm{~h}$ ). The tablets were crushed into powder, which was mixed with drinking water for rats. The treatment was carried out during the entire period of the experiment.

Voiding cystography was performed at 1, 3, and 6 months of the experiment, by injecting 3-5 $\mathrm{ml}$ of urographin into the cavity of bladder using a syringe. At the 6th month of the experiment, the levels of TGF- $\beta 1$, Ang II in the urine of animals were determined by ELISA and recalculated per mmol of creatinine determined in a specific urine portion ( $\mathrm{ng} / \mathrm{mmol}$ of creatinine). Removal of animals from the experiment was carried out after anesthesia by intraperitoneal administration of $0.4 \mathrm{ml}$ of $5 \%$ ketamine. Morphological studies were performed by macro- and microscopy of the renal-ureteral complexes obtained from individuals of the experimental groups and the control group. The material for histopathological research was biopsy specimens $(1 \times 1 \times 0.5 \mathrm{~cm})$ of the renal-ureteric complex obtained at necropsy of animals, previously fixed in $4 \%$ and $10 \%$ formalin solution for no more than 12 hours. Histopathological processing of material in biopsy specimens was carried out according to standard protocols of histological technology using routine (hematoxylineosin - GE) and special (picrofuksin according to Van-Gizon (VG), aniline blue according to Mason) coloring methods. The collection of material from rats of the control group was carried out in dynamics simultaneously with experimental animals (1 rat in 1,3 and 6 months).

The following morphological changes were taken for statistical analysis: cell infiltration, interstitial edema, and interstitial fibrosis. For the analysis of morphological data, an estimated ball scale was used, described by Rodrigues-Iturbe (2005), where 0 is unchanged, 1 is weak, 2 is moderate and 3 is pronounced changes (Figure 1) \& (Table 1). ${ }^{14}$ The program Statistica for Windows 6.0 was used for statistical processing of the obtained results with conducting correlation analysis. Significant differences were considered indicator sat $\mathrm{p}<0.05$ (Figure 2). After dissection at 1 month of experiment, animals from Group 1 with VUR had kidneys of normal size, capsule was shiny, pyelocaliceal segments slightly widened, with mild hyperemia of the cortical parenchyma and perirenal cellular tissue. Histologically we observed moderate polymorphic-cell inflammatory lymphoplasmacytic infiltration (Figure 3 (A)), slightly extended convoluted tubules, glomeruli were in most cases stored (Figure 3 (B), mild interstitial edema, and focal capillary hyperemia (Figure 3 (C). Minimal processes of sclerosis were revealed in the cortical area of the capsule of some glomerules and in glomerular basement membrane in convoluted periglomerular tubules (Figure 3 (D).

Animals with VUR of Group 2 group had the kidney slightly enlarged in size on the side of reflux, and we observed enlargement of pyelocaliceal segment and plethora of cortical zone and perirenal cellular tissue. The dissection also revealed translucent exudates in the abdomen of rat, also we established urothelial proliferationinpyelocaliceal segments (Figure 4 (A) and more expressed polymorphic cell infiltration (Figure 4 (B) and moderate collagenization of subepithelial zone. There was smoothness of papillary structures, expansion of the tubular portion of the nephron, moderate degeneration and necrosis of nephrothelial convoluted tubules. Simultaneously, we observed hypotrophy of arterial vessels with their moderate stenosis with more pronounced segmental glomerular basement membrane and tubule thickening (Figure 4 (C), there was hypotrophy of vessel walls in combination with slight perivascular sclerosis, cell infiltration and moderate hyperplasia withfibroblasts (Figure 4 (D), that were not observed in Group 1.

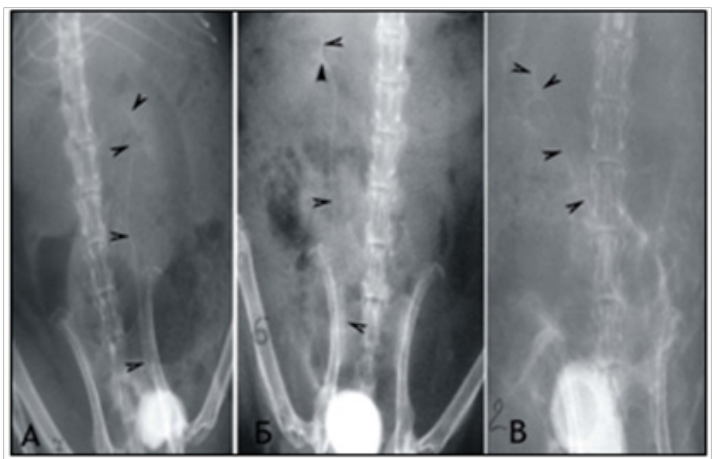

Figure I Cystography in rats. Cystic ureteral reflux of the III degree at the 6th month of the experiment:A) on the left in the individual of the Ist group; B) on the right of the individual 2 groups.

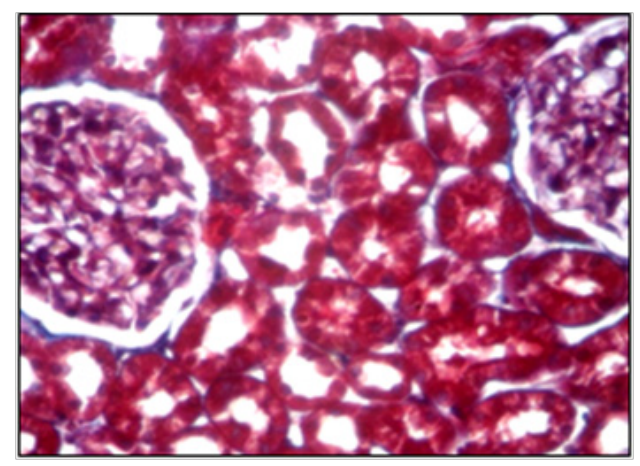

Figure 2 Microscopic aspect of the kidney of a rat from the control group at 6 months. Cortical zone of the kidney parenchyma, Mason aniline blue coloration about 20 oc. ten.

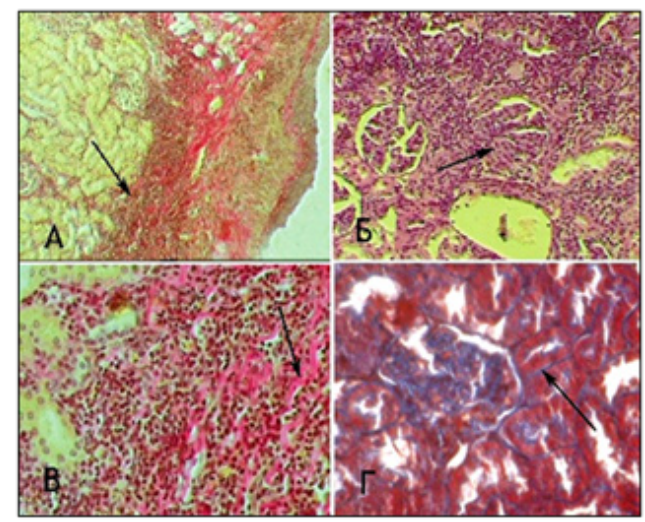

Figure 3 Microscopic changes in an individual from Group I with VUR at I month of the study. Painting of Van Gies on (A) x100, in Mason (B, D) $\times 100$. 
Table I Incidence and degree of VUR in the studied groups of rats

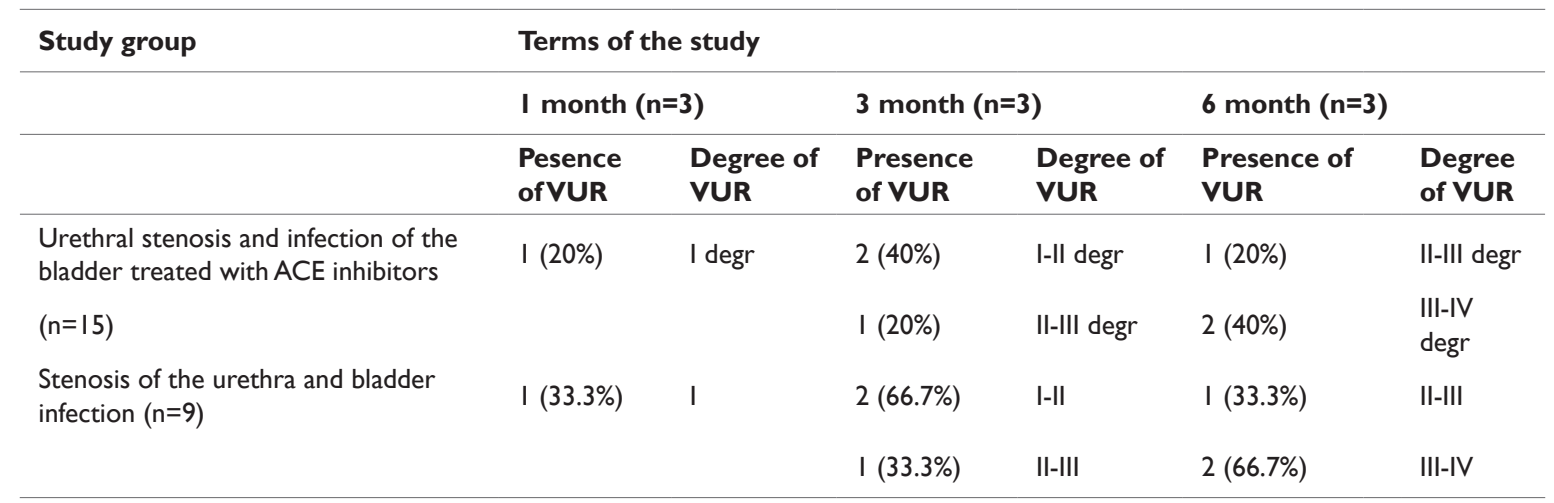

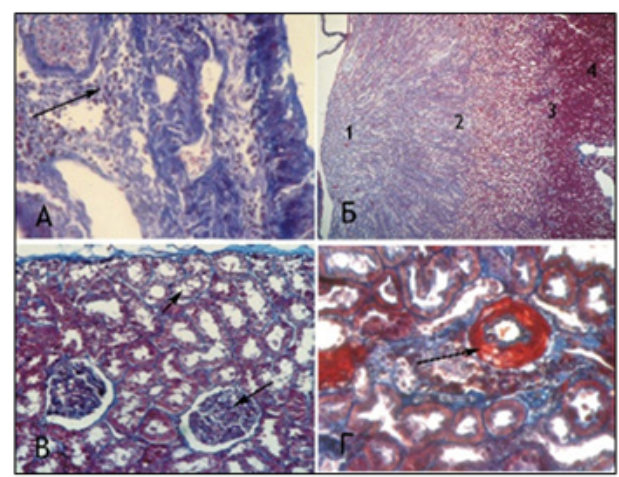

Figure 4 Microscopic changes in the kidneys of rats with VUR in Group 2 at I month of the study. Staining by Masson. xI00 (A, C, D), x 25 (B); Objective. 20. ocular 10 .

Figure 5 shows the comparative amounts of cellular infiltration levels, tubulointerstitial edema and sclerosis of renal tissues in rats of two groups after one month of experiment. The level of interstitial edema differed insignificantly in both groups, while the level of cellular infiltration and fibrosis of renal tissue was significantly lower in rats of group 1 than in animals of group $2(p<0,05)$. In individuals of group 1 after 6 months experiment the kidneys were slightly increased in size on the side of VUR, dark brown, shiny capsule, in some individuals with VUR capsules had areas of retraction. In 3/5 of individuals with VUR mucous membrane of the renal pelvis was swelling, also we revealed unexpressed focal sclerosis at the level of the cups. When revising of cup segments, we observed atrophy of papillary structures (Figure 6 (A), mild lymphocytic infiltration and slight intravascular stasis (Figure $6(\mathrm{~B}, \mathrm{C})$, focal thickening of the basement membrane and its hardening (Figure 6 (D). It should be noted that the processes of sclerosis were minimal.

In individuals of Group 2 2/3 (66.7\%) cases with VUR of III-IV degree kidneys were increased in volume by 1.5 -2 times, until reaching

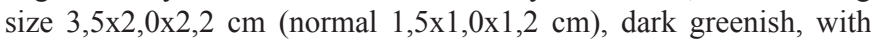
unclear contours lumpy; pelvis and calyx extension (hydronephrosis transformation). In one of cases kidneys were reduced in size and "whitish". We saw signs of atrophy of renal parenchyma, the thickness of which reached 0.1-0.3 mm (with normal thickness of 1.0-1.2 mm) and "sack" deformation of pelvis, wherein the lumen contained purulent masses. There was no differentiation between the cortex and medulla in all samples, with thinned parenchyma. In one of cases we saw kidney capsule with region of retraction. When stained by Mason it showed marked atrophy of nephrothelium with obliteration with hyaline cylinders (Figure 7 (A); expressed processes of sclerosis with polymorphocellular infiltration of the parenchyma with thickening of basement membrane of tubules and capsules glomeruli (Figure 7 (B) parallelly with interstitial fibrosis we observed severe lymphohistiocytic and plasmocitic infiltration, covering entire thickness of parenchyma, convergence and ischemic destruction of glomeruli, lymphohistiocytic infiltration and edema, collagenization of intracapsular space and hyalinosis of capillary loops (Figure7(C \& D). At the 6th month of the experiment, the level of interstitial edema did not differ in both groups ( $p>0.05)$, while cell infiltration and fibrosis in tubulointerstitium was significantly $(\mathrm{p}<0.01)$ higher in group 2 than in group 1 (Figure 8).

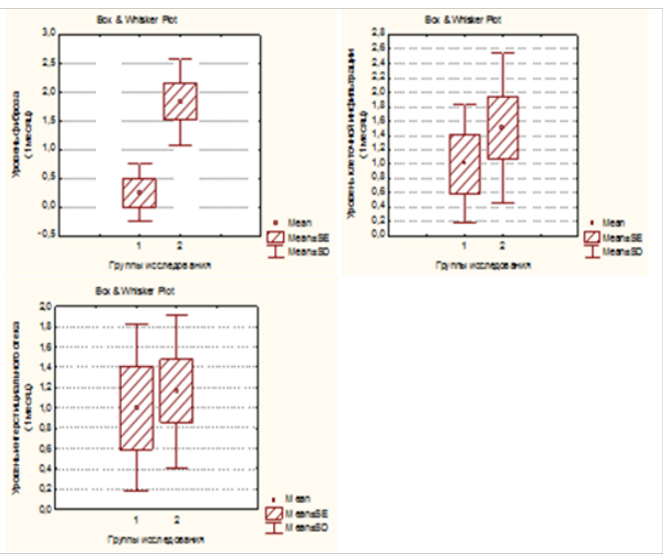

Figure $\mathbf{5}$ The level of tubulointerstitial edema, cellular infiltration and fibrosis of the renal parenchyma in rats of both groupsinmonths of experiment.

To determine the nephroprotective effect ofEnalapril on markers of sclerosis of renal tissue we examined excretion of profibrogenic factors with urine in both experimental groups at 6 months of the experiment and in the control group (Group 3, $\mathrm{n}=3$ ). Increased urinary excretion of TGF- $\beta 1$ was found in all specimens with the VUR compared with the control group $(\mathrm{p}<0,05)$. At the same time the animals in Group 2 had significantly higher levels of urinary excretion of TGF- $\beta 1$ as compared with Group 1 ( $p<0,001$, Figure 9). Ang II levels in urine specimens of Group2 with VUR not treated with Enalapril, was 2 fold higher than in the control group $(\mathrm{p}<0,05)$. In rats of Group 1 Ang II levels in urine were significantly lower than in Group $2(p<0,001)$ (Figure 10). Consequently, a significantly higher urinary levels of Ang II and, in particular, TGF- $\beta 1$ in rats not treated with an ACE 
inhibitor (Group 2) compared to the rats fed with Enalapril (group 1) for 6 months, indicating that one of the pathogenetic mechanisms of renoprotective action of ACE inhibitors is their inhibitory effect on the Ang II and indirectly on the TGF- $\beta 1$. The level of these profibrogenic factors in the urine can reflect the severity of sclerotic changes in the renal tissue. This is also confirmed by the presence in rats of Group 2astrong direct correlation between the levels of TGF- $\beta 1$ and Ang II, on the one hand, and the degree of sclerosis on the other hand $(r=0.67$ and $\mathrm{r}=0.78$, respectively, $\mathrm{p}<0.05$ ).

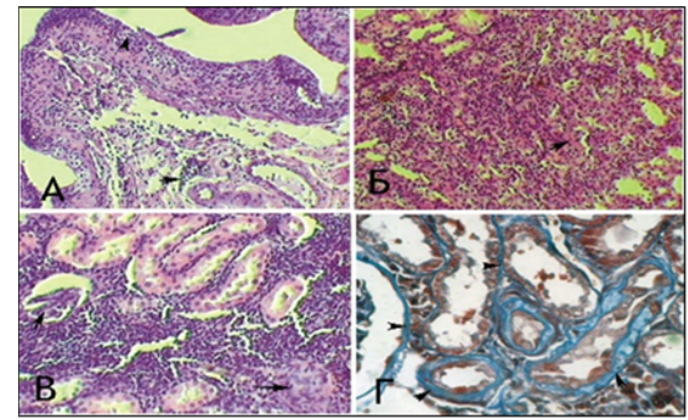

Figure 6 Microscopic changes in the structural tubulointerstitial component of kidneys in rats of group I withVURafter 6 months of experiment. HE staining (A.B.V) and Mason (D) Ob.40.ok.10 (B, D) Ob.20.ok. 10 (A, B)

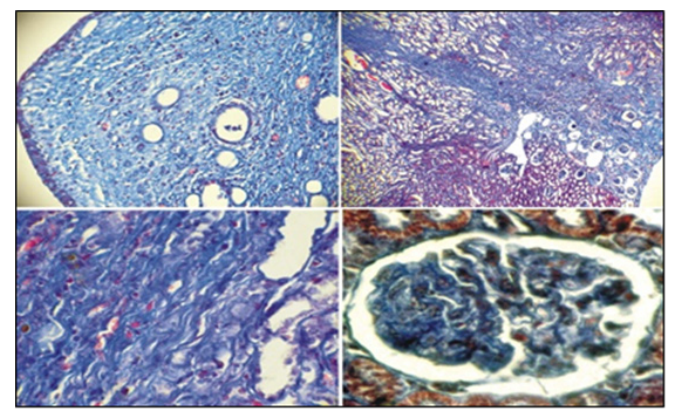

Figure 7 Sclerotic processes and collagen formation in the kidney parenchyma in rats of group 2 with VUR after 6 months of experiment. Staining by Mason. (A, B, C, D) Ob.2,5. oc. I0. (A, B, C) Ob.40.oc. 10 (D).
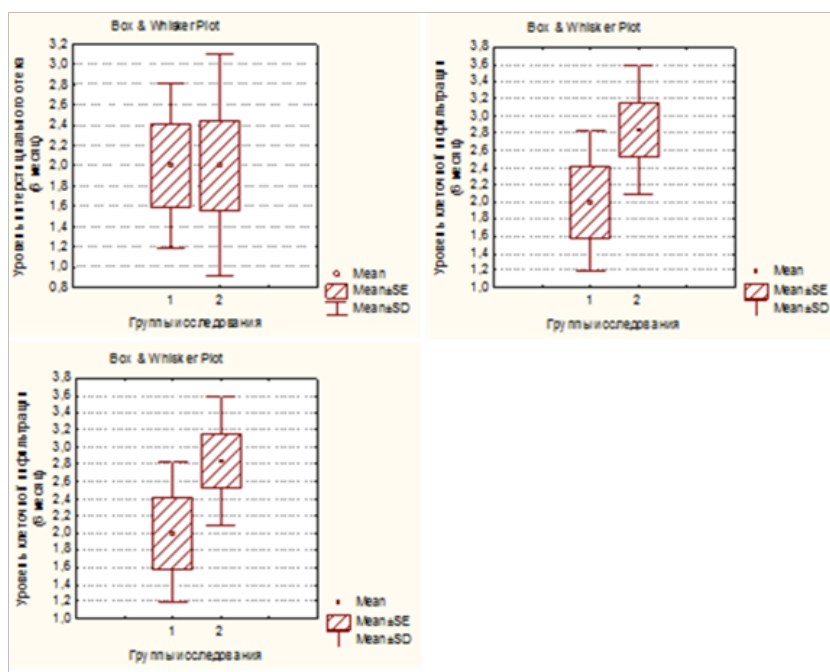

Figure 8 The level of tubulointerstitial edema, cell infiltration and rena parenchyma fibrosis in rats of both groups at the 6th month of the experiment.

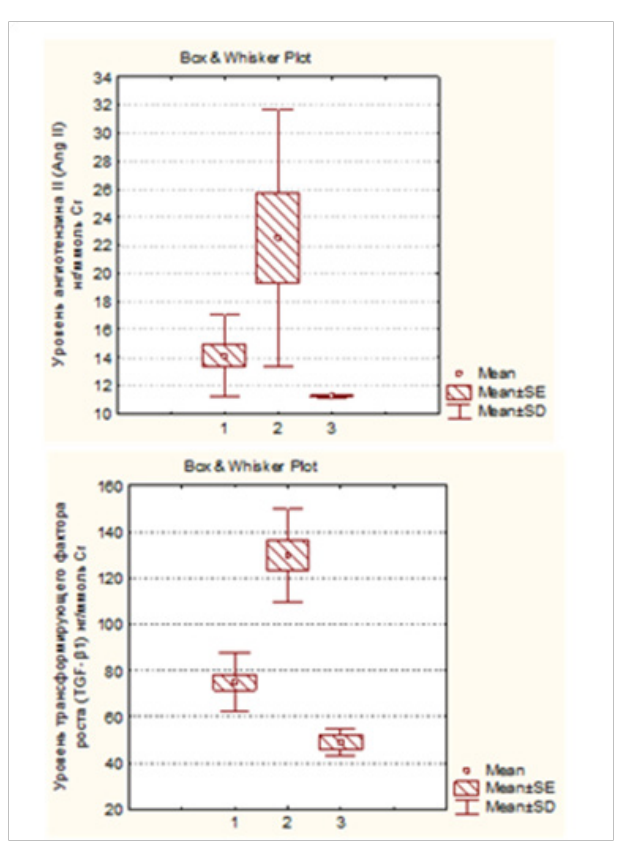

Figures 9 \& 10 The level of TGF- $\beta I$ and Ang II (ng/mmolofcreatinine) in the urine of rats, where Group I - individuals treated with Enalapril, Group 2 - the rat, not treated with Enalapril, 3 - control group.

\section{Discussion}

Since the first months of the experiment the rats of both groups, the vascular component characterized hypertrophy walls intrarenal arterioles, small intimal thickening, perivascular fibrosis, and hyperemia peritubular and glomerular capillaries in all samples, but more pronounced in 2 group not receiving enalapril, which characterizes development of local glomerular hypertension. Probably, the growth of pathological vascular changes is a result of direct exposure to bacterial and toxic factors in the intima of the latter with the development of dystrophic and ischemic damage, and also due to increase of hydronephrosis transformation nephron with the development of interstitial sclerosis, as has not been fixed obstruction, as evidenced by the prevalence of pathological changes in the observing the dynamics. Intensity of interstitial edema in the first month of the experiment was not significantly different between the groups $(p>0,05)$, although it had a tendency to grow to the 6-month experiment, with more pronounced in the second group of individuals. At the same time, on the first month of the experiment, the level of cellular infiltration in kidney parenchyma was significantly lower in Group 1 compared to Group $2(p<0,05)$. Rats not treated with enalapril (group 2 ) had a significantly more rapid formation of collagen fibers in the renal parenchyma when compared to group $1(p<0,05)$, which received an ACE inhibitor. Since the proximal tubules damaged by ischemia and increased hydrostatic pressure are the main source of growth factors, elevated levels of TGF- $\beta 1$ and Ang II in the urine in rats with VUR indicates hyper production of cytokines in the kidney. ${ }^{15,16}$ Given the identified correlation between the levels of profibrogenic factors in the urine of rats and investigated the severity of fibrosis in the tubulointerstitium, we can assume that TGF- $\beta 1$ and Ang II activate fibroblast proliferation with the growth of connective tissue in the kidneys, in agreement with data Liang $\mathrm{XL}$ et al., ${ }^{6}$ and Peter $\mathrm{J}$ et al., ${ }^{18}$. Our findings are consistent with the 
data presented by Hussein A et al., ${ }^{10}$ who identified the relationship between urohemodynamic disorder, urinary tract infection and the activity of TGF- $\beta 1$ in the blood. ${ }^{17,18}$

\section{Conclusion}

Thus, the results of morphological studies of the kidneys and profibrogenic factors in the urine in rats with simulated infected VUR, complicated by RN showed that early administration of ACE inhibitors for Ang II blockade and renoprotection prevented the development of severe nephrosclerosis, as well as in this group of rats was less pronounced degree of cell infiltration. This is probably due to the action of an ACE inhibitor both on the systemic and locally on intrarenal hemodynamics and on the reduction of the intrarenal production of Ang II and TGF- $\beta 1$, which ultimately leads to a decrease in intraglomerular capillary pressure and a decrease in fibrosis of the renal tissue, which is consistent with Kagami S et al., ${ }^{19}$ and Serlachius E et al. ${ }^{20}$ (1997). ${ }^{19,20}$ The levels of profibrogenic factors in the urine were significantly lower in the group that received Enalapril for 6 months. Based on the results obtained, ACE inhibitors reduce the formation of fibrous tissue in the kidneys by reducing the production of extracellular matrix and profibrogenic growth factors fibroblasts, but do not affect already formed nidi of sclerosis.

\section{Acknowledgments}

None.

\section{Conflicts of interest}

The author declares there is no conflict of interest.

\section{References}

1. Anichkova IV, Papayan AB. Reflux-nephropathy. Clinical Nephrology Pediatric SPb. St. Petersburg. 2008;452-457.

2. Sevgi Mir, Pelin Ertan, Nese Ozkayin et al. Risk Factors for Renal Scarring in Children with Primary Vesicoureteral Reflux Disease. Saudi J Kidney Dis Transpl. 2013;24(1):54-59.

3. Lakomova DY, Starodubova AV, AV Maximov Early diagnosis and prediction of renal scarring in children with vesicoureteral reflux. Bulletin of Medical Internet Conferences. 2012:2(1):27-28.

4. North American Pediatric Renal Transplant Cooperative Study (NAPRTCS). Annual reports. 2010. The EMMES Corporation, Rockville, MD. 2010. p. 1-102.

5. Silva AC, Flynn JT. The renin-angiotensin-aldosteron system in 2011: role in hypertension and chronic kidney disease. Pediatr Nephrol. 2012;27(10):1835-1845

6. Liang XL. Beyond Early Diagnosis: Prognostic Biomarkers for Monitoring Acute Kidney Injury. Hong Kong J Nephrol. 2010;12(2):45-49.
7. Chichuga EM, Nastausheva TL, Zvyagina TGF. Markers of chronic kidney disease in children with urinary tract obstruction or vezicoureteral reflux. Pediatric Pharmacology. 2015;12(4):407-413.

8. Urazaeva LI, Maksudova AN. Biomarkers of early kidney damage: Nephrology, the practice of medicine, innovative technologies in medicine. 2014;1:125-130.

9. Flown YY, Saganova ES, AV Smirnov. Biomarkers in the diagnosis of acute kidney injury. Nephrology. 2014;4(6):25-35.

10. Hussein A, Askar E, Elsaeid M, Schaefer F. Functional polymorphisms in transforming growth factor-beta-1 (TGFbeta-1) and vascular endothelial growth factor (VEGF) genes modify risk of renal parenchymal scarring following childhood urinary tract infection. Nephrol Dial Transplant. 2010;25(3):779-785

11. Chertin B, Rolle U, Cascio S. Upregulation of angiotensin II receptors in reflux nephropathy. Pediatr Surg. 2002;37(2):251-255.

12. Лопухин ЮМ. Экспериментальная хирургия. Medicine. 1971. p. 222.

13. Khalil A, Tullus K, Bakhiet M, et al. Angiotensin II type I receptor antagonist (Losartan) down regulates transforming growth factor- $\beta$ in experimental acute pyelonephritis. J Urol. 2000;164(1):186-191.

14. Rodriguez-Iturbe B, Quiroz Y, Shahrarami A, et al. Mycophenolemofetil ameliorates nephropathy in the obese Zucker rat. Kidney Int. 2005:68(3):1041-1047.

15. Morales MG, vazquez Y, Acuña MJ, et al. Angiotensin II-induced profibrotic effects require p38MAPK activity and transforming growth factor beta 1 expression in skeletal muscle cells. Int J Biochem Cell Biol. 2012;44(11):1993-2002.

16. Paltiel HJ, Mulkern RV, Perez-Atayde A, et al. Effect of chronic, lowpressure, sterile vesicoureteral reflux on renal growth and function in a porcine model: a radiologic and pathologic study. Radiology. 2000;217(2):507-515.

17. Liang XL. Beyond Early Diagnosis: Prognostic Biomarkers for Monitoring Acute Kidney Injury. Hong Kong Journal of Nephrology. 2010;12(2):4549.

18. Peter J Margetts, Catherine Hoff, limin Liu, et al. Transforming growth factor $\beta$-induced peritoneal fibrosis is mouse strain dependent. Nephrol Dial Transplant. 2012;28(8):2015-2027.

19. Kagami S, Border WA, Miller DE, et al. Angiotensin II stimulates extracellular matrix protein synthesis through induction of TGF- $\beta 1$ expression in rat glomerular mesangial cells. $J$ Clin Invest. 1994;93(6):2431-2437.

20. Serlachius E, Sundelin B, Eklof A. Pyelonephritis provokes growth retardation and apoptosis in infant rat renal cortex. Kidney Int. 1997;51(6):1855-1862. 